\title{
ANALYSIS OF THE IGNITION CONTROL IN THE HCCI SYSTEM USING DOSE OF MONOATOMIC INERT GAS
}

\author{
Stefan Postrzednik, Grzegorz Przybyła, Zbigniew Żmudka \\ Silesian University of Technology \\ Institute of Thermal Technology \\ Konarskiego Street 22, 44-100 Gliwice, Poland \\ tel.: +48 32 2371231, fax: +48322372872 \\ e-mail: stefan.postrzednik@polsl.pl
}

\begin{abstract}
The subject of the analysis presented in the paper is the idea and implementation method of the ignition point of homogeneous air-fuel mixture, used in the IC engine with HCCI system. Autoignition of the combustible mixture can occur only upon reaching the characteristic self-ignition temperature $T_{z}$. It should take place during (near end) the load compression. In order to achieve the fuel mixture ignition temperature $T_{z}$ in the system, usually it is proposed adjusting the engine compression ratio, $\boldsymbol{\varepsilon}$. However, it is quite cumbersome especially in case of frequent changes of engine load (there are also difficulties with the precise advance angle adjusting of fuel mixture ignition). According to our conception, the other method of autoignition adjustment can be successfully applied. The essence of the new idea consists in proper selection, according to engine load, of the exponent $\kappa$ of charge polytropic compression. Introducing the defined dose of monoatomic gas (e.g. argon Ar, $\kappa \approx 1.667)$ to air-fuel mixture, the higher values of the polytropic compression exponent $\kappa$ of working medium can be achieved. It will result in significant increase of the temperature rise $\Delta T_{\max }$ of the compressed charge and accordingly progressive increase of the advance angle of the mixture ignition.
\end{abstract}

Keywords: IC engine, HCCI system, ignition control, charge compression

\section{Introduction - subject of the analysis}

Progress and further development of heat engines with internal combustion largely involves improvement of physicochemical parameters of processes occurring in the system, which also refers to the way of ignition and next combustion of the prepared mixture. Very high expectations are associated with the mastery and the implementation of ignition and combustion, known as the HCCI (Homogeneous Charge Compression Ignition) system [3, 5].

The main idea of this system consists in controlled auto-ignition of the homogeneous air-fuel mixture (instead of fuel injection control - as in conventional diesel engines or ignition advance angle - as in spark-ignition engines). Fuel injection into the combustion chamber occurs at the end of the first part of the charge compression stroke. Combustible mixture is formed, which next ignites spontaneously in all its volume, by the end of compression stroke (before top death centre).

Combustion also continues through part of expansion (work) stroke until the mixture burned out completely. The course of the whole phenomenon is conditioned mainly by the chemical kinetics of fuel oxidation. An important role plays the ignition temperature $T_{z}$ of combustible mixture, above which, the probability of ignition is very high (almost certain).

Both rich (near stoichiometric), and more lean mixtures can be burnt, that enables governing of engine load and efficient programming of the combustion process (rate of pressure increase) in a cylinder. However, the choice of fuel type (special, synthetic fuel can also be used) is also very important $[4,6]$.

The HCCI combustion is a rapid process, therefore, to limit the pressure rise rate, the lean mixtures and exhaust gas recirculation can be applied, which generally slows down the 
combustion process. Exhaust gas recirculation plays an important role in the selection of the ignition point of combustible mixture and next influences the kinetics parameters of oxidation of fuel [1], so the whole combustion phasing in the system.

Developed until now and proposed technical solutions of HCCI technology are still imperfect, mainly in the range of the combustion process controlling. Engines should be designed with the possibility of the compression ratio adjustment, the independent valves actuating system (setting of opening, closing angles and the lift of valves), selection of the exhaust gas recirculation (EGR). However, the fundamental problems associated with the possibility of full and effective engine management using HCCI system - mainly during start-up and various loads - have not been clearly and satisfactorily solved so far.

Research on HCCI combustion systems are widely performed and are inspired by the advantages of this system, which include - reduction of emissions of harmful substances (primarily nitrogen oxides $\mathrm{NO}_{\mathrm{x}}$ ), - diminishing of fuel consumption (improving of energy and economic efficiency of the engine), - possibility of combustion of different (conventional, gas, alternative) fuels $[2,3,5]$.

The analysis presented in this paper deals with a different, original way of the ignition point control of a homogeneous fuel mixture, used in the HCCI system.

The essence of the new idea consists in applying a specific dose of monoatomic gas (so-called noble gas, such as argon Ar) into the charge accumulated in cylinder. This gas will not participate directly in the combustion process but due to the highest ratio $\kappa \approx 1.667$ (ratio of specific heat capacities $\left(\mathrm{c}_{\mathrm{p}} / \mathrm{c}_{\mathrm{v}}\right)$, isentropic exponent $\left.\kappa\right)$, will efficient influence the temperature level at the end of the charge compression in the engine cylinder, depending on its concentration. Self-ignition of the homogeneous combustible mixture (HCCI system) can succeed reaching the characteristic, spontaneous ignition temperature $\mathrm{T}_{\mathrm{z}}$, which should occur before the end of the charge compression.

Thanks to proposed measure, the self-ignition temperature of the mixture will be attained at any engine operating conditions even at starting.

\section{Concept and basic elements of ignition control in the HCCI system}

According to the presented proposal - both an increase of the compression ratio $\varepsilon$ - and a growing of the monoatomic gas content $\mathrm{zl}$ in the charge, - contribute to rise of the temperature $\mathrm{T}_{2}$ of the charge at the end of its compression.

However, the control (through the dose) of the noble gas content in the charge should be practically much simpler to implement, than changing the engine compression ratio $\varepsilon$. A preliminary, simplified analysis presented in the paper confirms this assumption.

Assuming an isentropic course of the charge compression, the temperature increase $\Delta \mathrm{T}(\alpha)$, above the temperature $\mathrm{T}_{0}$ at the beginning of compression (the ideal gas model), is:

$$
\Delta \mathrm{T}(\alpha)=\mathrm{T}_{0} \cdot\left[\left(\frac{\mathrm{V}_{0}}{\mathrm{~V}(\alpha)}\right)^{(\kappa-1)}-1\right]
$$

where:

$$
\Delta \mathrm{T}(\propto)=\mathrm{T}(\propto)-\mathrm{T}_{0}, \mathrm{~V}_{\mathrm{k}} \leq \mathrm{V}(\propto) \leq \mathrm{V}_{0},
$$

and:

$\alpha-$ crank angle,

$\kappa=(\mathrm{cp} / \mathrm{cv})-$ specific heat ratio of the charge,

$\mathrm{V}(\alpha) \quad-$ current volume of the cylinder (charge),

$\mathrm{T}(\alpha) \quad$ - current temperature of the charge.

The maximum value of the temperature rise $\Delta \mathrm{T}_{\max }$ of the compressed charge is: 


$$
\Delta \mathrm{T}_{\max }=\mathrm{T}_{0} \cdot\left(\varepsilon^{(\kappa-1)}-1\right), \quad \text { and }: \varepsilon=\left(\frac{\mathrm{V}_{0}}{\mathrm{~V}_{\mathrm{k}}}\right),
$$

where:

$\varepsilon-$ compression ratio,

$\mathrm{V}_{\mathrm{k}}$ - minimum volume of the cylinder (combustion chamber).

To occur the auto-ignition of the compressed combustible mixture, the adequate temperature increase must be achieved, amounting to:

$$
\Delta \mathrm{T}_{\mathrm{z}}=\mathrm{T}_{\mathrm{z}}-\mathrm{T}_{0} \text {, with the condition: } \Delta \mathrm{T}_{\mathrm{z}} \leq \Delta \mathrm{T}_{\max },
$$

where $\mathrm{T}_{\mathrm{z}}-$ self-ignition temperature of the mixture prepared in the cylinder.

In order to satisfy inequality (4) and to achieve the auto-ignition temperature $T_{z}$ of the fuel mixture, an adequate adjustment of the engine compression ratio $\varepsilon$ is usually proposed, according to (3). However, it is quite cumbersome and ineffective control procedure of the system, especially for frequent changes of an engine load (there are also difficulties with the precise regulation of the ignition advance angle). Specific heat ratio $\kappa$, in this case as exponent of the isentropic charge compression (e.g. for air as 2-atom ideal gas) equals $\kappa=7 / 5=1.4$.

Exhaust gas recirculation (combustion products have lower values of specific heat ratio $\kappa<1.4$ ) contributes to decrease of the value of the exponent $\kappa$ of the isentropic charge compression (presence of carbon dioxide $\mathrm{CO}_{2}$ and water vapour $\mathrm{H}_{2} \mathrm{O}$ ) and also depression of the temperature after the charge compression. But on the other hand, it can be compensated by the relatively high enthalpy (thus the temperature) of the recirculated exhaust gases.

The different method of auto-ignition adjustment can be successfully applied. The essence of the method consists in the proper adjustment of the isentropic exponent (occurring in equations (1) and (3)) of the charge compression according to an engine load.

Adding to the fuel mixture a defined dose of monoatomic gas (e.g. argon, Ar, $\kappa=5 / 3 \approx 1.667$, $\mathrm{M} \approx 40 \mathrm{~kg} / \mathrm{kmol}$ ), higher values of the isentropic exponent $\kappa$ of the charge compression can be effectively achieved which, according to equation (3), will result in a significant increase of the temperature rise $\Delta \mathrm{T}_{\max }$ of the compressed charge.

The influence of the engine compression ratio $\varepsilon$ (for values: $8,10,12$ ) and the content $\mathrm{z}_{1}$ of the monoatomic gas (argon) in the mixture with diatomic gas (air, $\kappa=1.4)$ on the relative value of the charge temperature $\mathrm{T}_{2}$ achieved at the end of the compression (GMP) has been initially analysed. Test calculations were carried out and the results are presented in the Fig. 1.

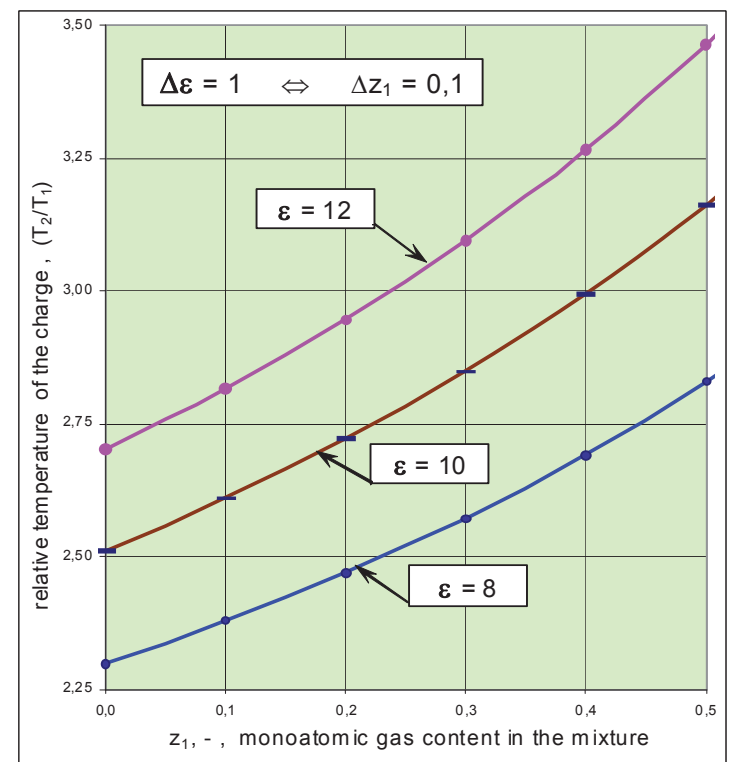

Fig. 1. Influence of compression ratio and content of the monoatomic gas on charge temperature after compression 
Both an increase of the compression ratio $\varepsilon$ and an increase of the monoatomic gas content $\mathrm{zl}$ in the charge lead to raise of the temperature $T_{2}$ at the end of charge compression, which can be observed in the Fig. 1.

One can make the estimated assumption that the change of the content of monoatomic gas by $\Delta \mathrm{z}_{1}=0.1$ gives a result comparable with the change of compression ratio by $\Delta \varepsilon=1$. However, the control of the monoatomic gas content (dose demand) in the charge appears practically much simpler to implement than changing the engine compression ratio $\varepsilon$. Apart from control of the temperature increase $\Delta \mathrm{T}_{\max }$ of the compressed charge, proposed method can also be used in the algorithm of selection of the ignition advance angle in the HCCI system.

Equating the required temperature increase $\Delta \mathrm{T}_{\mathrm{z}}$ - the dependence (4), conditioning the autoignition of the compressed fuel mixture, with the instantaneous temperature increase $\Delta \mathrm{T}(\alpha)$ formula (1), towards the required relationship relating to the current ignition angle $\alpha_{z}$ :

- it can be achieved:

$$
\Delta \mathrm{T}_{\mathrm{z}}=\Delta \mathrm{T}\left(\alpha_{\mathrm{z}}\right),
$$

$$
\Delta \mathrm{T}_{\mathrm{z}}=\mathrm{T}_{0} \cdot\left[\left(\frac{\mathrm{V}_{0}}{\mathrm{~V}\left(\alpha_{\mathrm{z}}\right)}\right)^{(\kappa-1)}-1\right]
$$

at:

$$
\Delta \mathrm{T}_{\mathrm{z}}=\mathrm{T}_{\mathrm{z}}-\mathrm{T}_{0}, \Delta \mathrm{T}_{\mathrm{z}} \leq \Delta \mathrm{T}_{\max },
$$

from this, the angle $\alpha_{z}$ can be determined, at which the auto-ignition of homogeneous mixture occurs.

The function $\mathrm{V}(\alpha)$ describing the instantaneous cylinder displacement (charge volume) can be expressed by relation:

$$
\frac{\mathrm{V}(\alpha)}{\mathrm{V}_{0}}=\frac{1+(\varepsilon-1) \cdot \mathrm{F}(\sigma, \alpha)}{\varepsilon}
$$

where the auxiliary function $\mathrm{F}(\sigma, \alpha)$ describes the relationship:

$$
\mathrm{F}(\sigma, \alpha)=\frac{1}{2}\left[(1-\cos (\alpha))+\frac{1}{\sigma} \cdot\left(1-\sqrt{\left[1-\sigma^{2} \cdot \sin ^{2}(\alpha)\right]}\right)\right],
$$

and $\sigma=(\mathrm{R} / \mathrm{L})$ - means the ratio of crank $(\sigma \approx 0.1-0.3)$,

where:

L - length of a connecting rod,

$\mathrm{R}$ - radius of a crank.

Typical shapes of piston way function $\mathrm{F}(\sigma, \alpha)$, for selected parameters $\sigma$, are shown in the Fig. 2 .

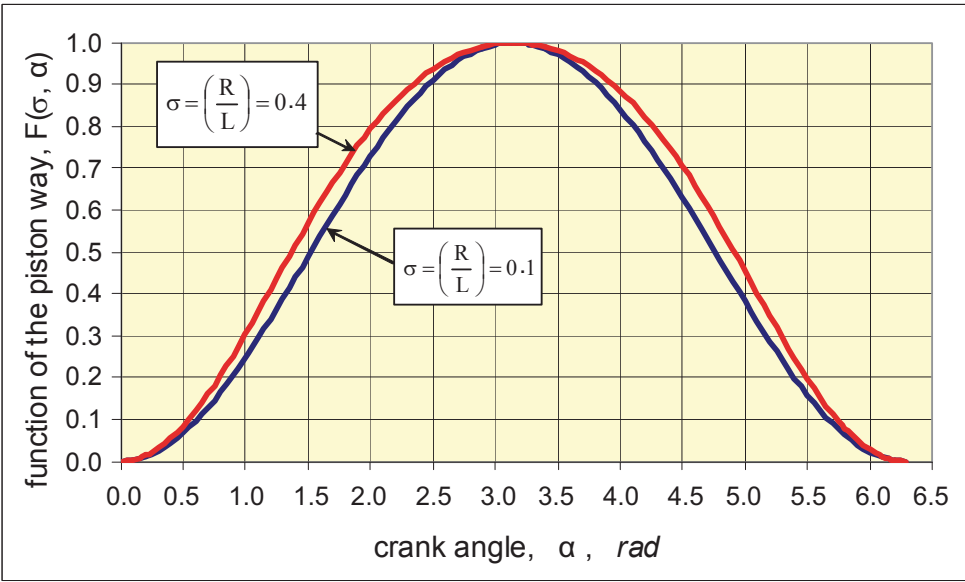

Fig. 2. The function $F(\sigma, \alpha)$ as the way of the piston in the cylinder 
After inserting the formula (8) in the equation (6) it is obtained:

$$
\left(\frac{\Delta \mathrm{T}_{\mathrm{z}}}{\mathrm{T}_{0}}\right)=\left[\left(\frac{\varepsilon}{1+(\varepsilon-1) \cdot \mathrm{F}\left(\sigma, \alpha_{\mathrm{z}}\right)}\right)^{(\kappa-1)}-1\right], \pi<\alpha_{\mathrm{z}} \leq 2 \pi,
$$

from this, using the auxiliary equation (9), one determines the searched angle $\alpha_{z}$.

The parameter $\kappa$, occurring in the above relationships, is the substitute (for the current composition of the charge) specific heat ratio (isentropic exponent) of the working medium, containing also the monoatomic gas (e.g. argon, Ar, $\mathrm{z}_{\mathrm{Ar}}$ ).

To find values of this substitute parameter $\kappa$, the following formula should be used:

$$
\left(\frac{1}{\kappa-1}\right)=\sum_{i=1}^{n}\left(\frac{z_{i}}{\kappa_{i}-1}\right) \text {. }
$$

For illustration, treating the charge as a two-component mixture: 1 - air (nitrogen $\mathrm{N}_{2}$, oxygen $\mathrm{O}_{2}, \kappa_{1}=7 / 5$ ) and 2 - argon (with the molar fraction $\mathrm{z}_{\mathrm{Ar}}$ and $\kappa_{2}=5 / 3$ ), the following expression is obtained:

$$
\kappa=1+\frac{2}{5-2 \cdot \mathrm{z}_{\mathrm{Ar}}}
$$

and accordingly the relation (10) takes the form:

$$
\left(\frac{\Delta \mathrm{T}_{\mathrm{z}}}{\mathrm{T}_{0}}\right)=\left[\left(\frac{\varepsilon}{1+(\varepsilon-1) \cdot \mathrm{F}\left(\sigma, \alpha_{\mathrm{z}}\right)}\right)^{\left(\frac{2}{5-2 \cdot \mathrm{z}_{\mathrm{Ar}}}\right)}-1\right] .
$$

According to the formulas (3) and (13), the maximum temperature increase $\Delta \mathrm{T}_{\max }$ is obtained at $\alpha_{\mathrm{z}, \max }=2 \pi$ for different argon content $\mathrm{z}_{\mathrm{Ar}}$ in the charge, so it amounts to:

$$
\left(\frac{\Delta \mathrm{T}_{\max }}{\mathrm{T}_{0}}\right)=\left[\left(\frac{\varepsilon}{1}\right)^{\left(\frac{2}{5-2 \cdot \mathrm{z}_{\mathrm{Ar}}}\right)}-1\right] \text {. }
$$

The determined relationship (13) is a significant correlation between the basic parameters of HCCI mixture auto-ignition system; - especially between self-ignition temperature $\mathrm{T}_{\mathrm{z}}$ and required argon content $z_{\mathrm{Ar}}$ in the charge as well as the angle of crankshaft $\alpha_{\mathrm{z}}$ at the moment of the charge self-ignition $\left[\left(2 \pi-\alpha_{z}\right)\right.$ - is the ignition advance angle].

For illustration, the function of the charge temperature $T(\alpha)$ during the compression in the cylinder, for the compression ratio $\varepsilon=10$, with different molar fractions $\mathrm{z}_{\mathrm{Ar}}$ of argon in the charge was analysed. The dependence resulting from the formulas (8), (13) describes the relative values of the instantaneous temperature $\left[\mathrm{T}(\alpha) / \mathrm{T}_{0}\right]$ of the charge and can be expressed as:

$$
\left(\frac{\mathrm{T}(\alpha)}{\mathrm{T}_{0}}\right)=\left(\frac{\varepsilon}{1+(\varepsilon-1) \cdot \mathrm{F}(\sigma, \alpha)}\right)^{\left(\frac{2}{5-2 \cdot \mathrm{z}_{\mathrm{Ar}}}\right)} .
$$

The obtained results, comprising the final part (for the crank angle $330{ }^{\circ} \mathrm{CA} \leq \alpha \leq 360{ }^{\circ} \mathrm{CA}$ ) of the charge compression (for the engine compression ratio $\varepsilon=12$, parameter $\sigma=0,25$ and argon molar fractions $0,0 \leq \mathrm{z}_{\mathrm{Ar}} \leq 0,30$ in the mixture) are shown in the Fig. 3. For higher argon contents $\mathrm{z}_{\mathrm{Ar}}$ the temperature of the charge gets respectively higher, but the extremum of the temperature $T(\alpha)$ is always in the top dead centre that is for $\alpha_{\mathrm{g}}=360^{\circ} \mathrm{CA}$ and equals $\mathrm{T}_{\max }\left(\mathrm{z}_{\mathrm{Ar}}\right)$.

The requested situations, when the value of auto-ignition temperature $T_{z}$ of the fuel mixture will be below the maximum temperature $\mathrm{T}_{\max }\left(\mathrm{z}_{\mathrm{Ar}}\right)$, can be achieved properly adjusting parameters of the system. Then a prospect of the self-ignition appears automatically, of course before a piston reaches top death centre $\left(\alpha_{g}=360^{\circ} \mathrm{CA}\right)$. 


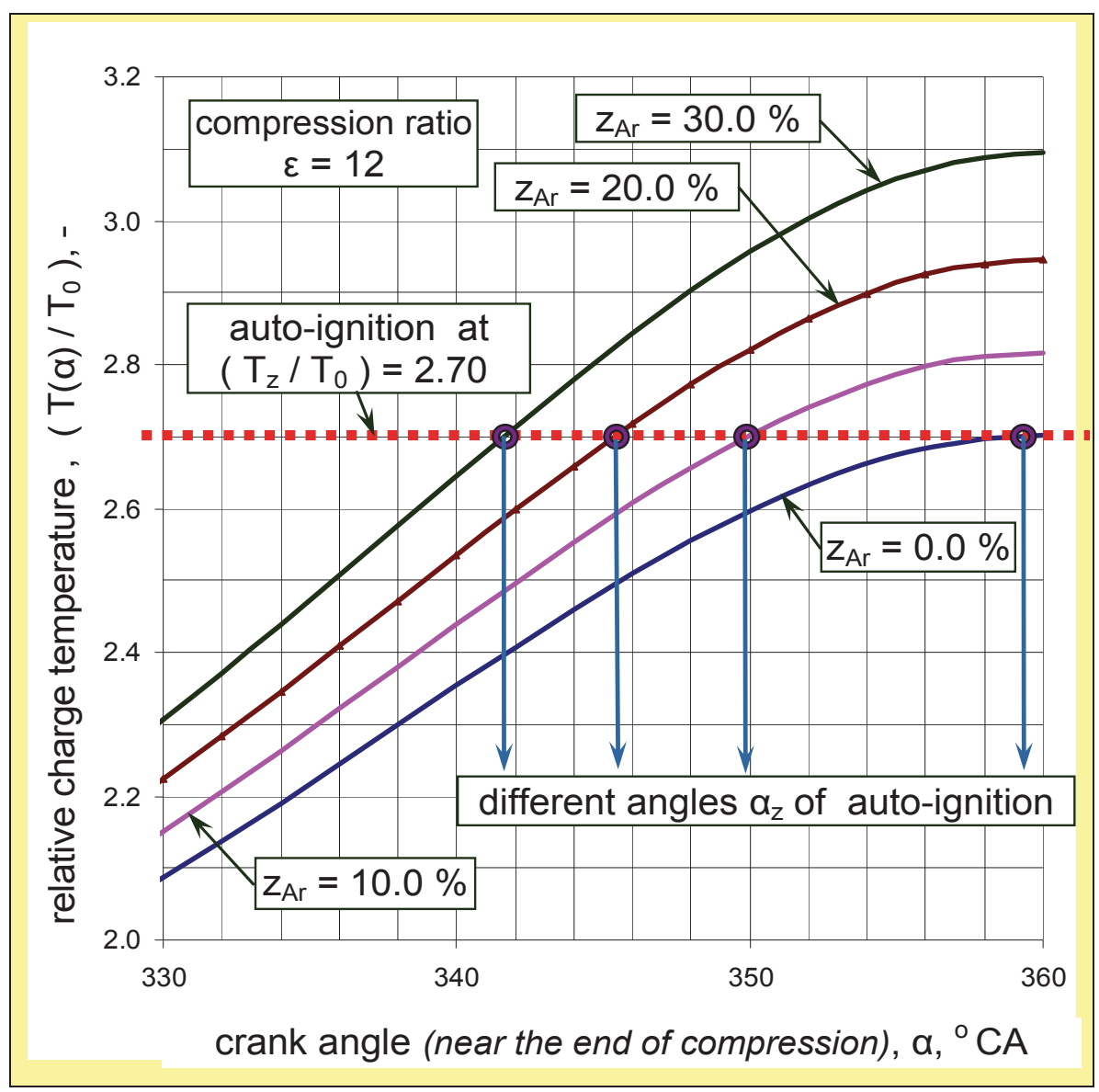

Fig. 3. Selection and control of the ignition advance angle for the HCCI system

For this purpose, the exemplary, relative value of the fuel mixture self-ignition temperature $\left(\left[\mathrm{T}_{\mathrm{z}} / \mathrm{T}_{0}\right]=2.70\right)$ is also presented.

Different values of the ignition advance angle $\left(2 \pi-\alpha_{z}\right)$ can be achieved depending on the molar fraction of the argon $\mathrm{z}_{\mathrm{Ar}}$ in the charge. For the taken data, the obtained values average:

\begin{tabular}{|c|c|c|c|c|c|}
$\mathrm{z}_{\mathrm{Ar}},-$ & 0.0 & 0.05 & 0.1 & 0.2 & 0.3 \\
\hline$\left(2 \pi-\alpha_{\mathrm{z}}\right),{ }^{\mathrm{o}} \mathrm{CA}$ & 0.7 & 6.8 & 10.6 & 14.5 & 18.4
\end{tabular}

This is the basic function of the new algorithm used for the regulation of the internal combustion engine operating in HCCI mode. Replacement of the nitrogen N2 in the charge (oxycombustion system) by the monoatomic gas would be considerable matter.

\section{Preliminary experimental tests}

In order to confirm the impact of presence of the argon $\left(\mathrm{Ar}, \mathrm{z}_{\mathrm{Ar}}\right)$ in the charge on the course of the compression (pressure $p(\alpha)$ and temperature $T(\alpha)$ changes), the adequate tests were carried out using the indication technique.

The basic data of the tested spark ignition engine:

- number of cylinders: 3, - displacement volume of one cylinder: $0.265 \mathrm{dm}^{3}$,

- compression ratio: 9.3, - ratio of a crank: 0.321.

The molar fraction of the argon in the charge (at $293 \mathrm{~K}$, at the pressure of $100 \mathrm{kPa}$ ) was changed within the range $0.0 \leq \mathrm{z}_{\mathrm{Ar}} \leq 0.19$.

The pre-heated (coolant temperature averaged $84^{\circ} \mathrm{C}$ ), tested engine was driven by the external source (electric motor - generator) with the speed $1500 \mathrm{rpm}$. 
Instantaneous values of the pressure $\mathrm{p}(\alpha)$ in the cylinder were recorded using the indication measurement system. Obtained results are presented in the Fig. 4.

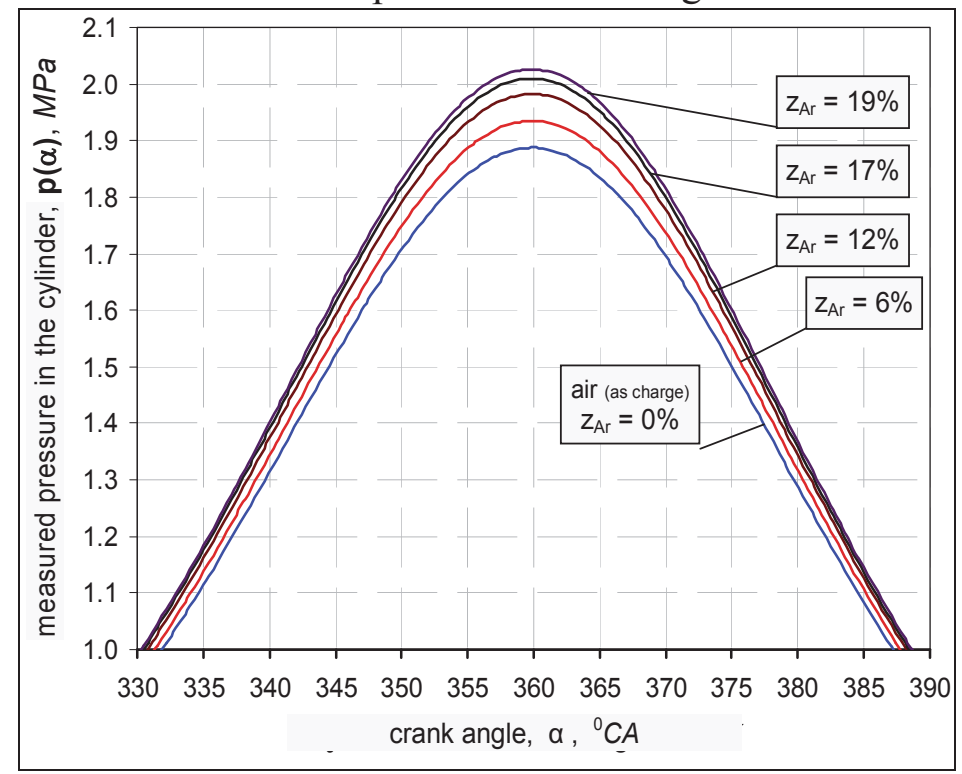

Fig. 4. Influence of the argon content in the charge on the pressure in the cylinder

Then, the temperature function $\mathrm{T}(\alpha)$ was determined using the ideal gas low:

$$
\mathrm{T}(\alpha)=\mathrm{T}_{0} \cdot \frac{\mathrm{p}(\alpha)}{\mathrm{p}_{0}} \cdot \frac{\mathrm{V}(\alpha)}{\mathrm{V}_{0}},
$$

and taking into account the relations (8), (9), the following formula was finally used:

$$
\mathrm{T}(\alpha)=\mathrm{T}_{0} \cdot \frac{\mathrm{p}(\alpha)}{\mathrm{p}_{0}} \cdot \frac{1+(\varepsilon-1) \cdot \mathrm{F}(\sigma, \alpha)}{\varepsilon}
$$

The determined temperature functions $T(\alpha)$ are shown in the Fig. 5.

Presented in the Fig. 4 and the Fig. 5 results of the SI engine indication investigations expressly confirm the possibility of practical use of the proposed new method of control of the HCCI process in combustion engine.

Further investigation will include test procedures with an engine operating "hot", that is normally working with full application of HCCI system.

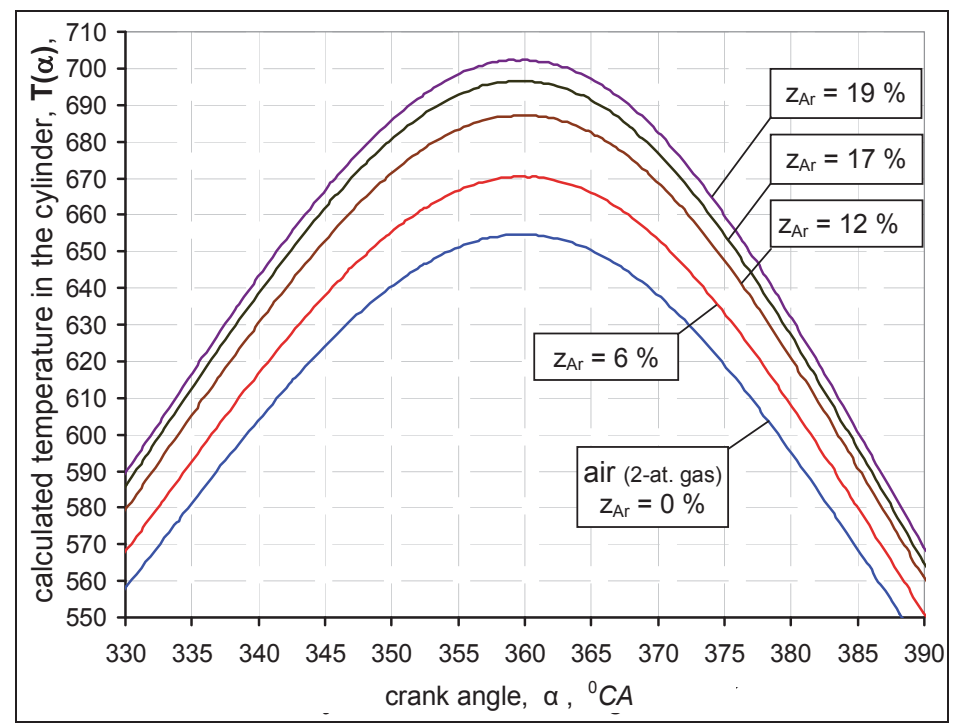

Fig. 5. Influence of the argon content in the charge on the temperature in the cylinder 


\section{Conclusion}

The paper presents an original way of the fuel mixture ignition control developed for the HCCI system. The proposed method consists in adding a strictly determined specific dose of monoatomic (noble) gas into the engine charge. The elaborated theoretical and experimental results confirm the theses stated. Both growth of the engine compression ratio $\varepsilon$ and increase of the monoatomic gas content in the charge contribute to raise of the temperature of the working medium at the end of its compression. By that way, auto-ignition of the fuel mixture can be realised securely and the autoignition advance angle can be adjusted in accordance with the needs (a load and an engine speed).

The fact of the matter is control of the molar fraction of the monoatomic gas in the charge should be practically much easier to implement than regulation of the engine compression ratio $\varepsilon$. This is significant advantage of the proposed method. Prospect of replacing the nitrogen $\mathrm{N}_{2}$ by the monoatomic gas in the charge would be fundamental matter.

\section{References}

[1] Ferguson, C. R., Internal Combustion Engines, Applied Thermo-Sciences, John Wiley \& Sons, Inc., 1986.

[2] Hany, A., Mohsen, A., Selim, A., Osama, A., NO emission control in SI engine by adding argon inert gas to intake mixture, Energy Conversion and Management 50, pp. 2699-2708, 2009.

[3] Johansson, R., Blom, D., Karlsson, M., Ekholm, K., Tunestal, P., HCCI Engine Modelling and Control using Conservation Principles, Society of Automotive Engineers, 01, p. 0789, 2008.

[4] Merkisz, J., Ekologiczne aspekty stosowania silników spalinowych, Wydawnictwo Politechniki Poznańskiej, Poznań 1994.

[5] Postrzednik, S., Żmudka, Z., Termodynamiczne oraz ekologiczne uwarunkowania eksploatacji tłokowych silników spalinowych, Wydawnictwo Politechniki Śląskiej, Gliwice 2007.

[6] Postrzednik, S., Żmudka, Z., Eksploatacyjne uwarunkowania wykorzystania eko-obiegu silnika spalinowego, IV. International Congress on Combustion Engines, PTNSS, 2011-SC-099. 